\title{
Advances in Multi-Beam and Multi-Ion FIB-SEM for 3D Correlative Microscopy
}

B. Winiarski ${ }^{1,2 *}$, R. Geurts ${ }^{3}$, S.J. Randolph ${ }^{4}$, R. Gannon ${ }^{4}$, G. Pyka ${ }^{1}$, T. Varslot ${ }^{5}$ and P.J. Withers ${ }^{2}$

1. Thermo Fisher Scientific, V.Pecha 12, Brno 627 00, Czech Republic

2. Henry Royce Institute, School of Materials, The University of Manchester, M13 9PL, U.K.

3. Thermo Fisher Scientific, Achtseweg Noord 5, 5651 GG Eindhoven, Netherlands

4. Thermo Fisher Scientific, 5350 NE Dawson Creek Dr, Hillsboro, OR 97124, USA

5. Thermo Fisher Scientific, Stiklestadveien 1, 7041 Trondheim, Norway

*Corresponding author: Bartlomiej.Winiarski@thermofisher.com

Three-dimensional (3D) correlative microscopy/tomography (CMT) in materials [1-3] and life sciences uses various versatile micro/nano $\mathrm{x}$-ray computed tomography scanners, optical microscopes, dual beam microscopes, transmission electron microscopes (TEM), nano computed tomography (CT) in TEM and SEM, etc. CMT for the same region of interest and at different length scales allows spatial and temporal registration in two and three dimensions of many imaging modalities. For example, visible light imaging, electron and cathodoluminescence imaging, ion microscopy, EBSD and EDS analytics, $x$-ray tomography, magnetic resonance imaging, Raman, atomic force microscopy, etc. information may be merged together bringing a new insight on the same region of interest.

The field of correlative microscopy is broad and relatively young, thus the capabilities of the various approaches have yet to be fully explored, and uncertainties remain when considering the best choice of strategy and workflow for the correlative experiment. Recent developments in microscopy and tomography techniques have been instrumental to progress in the materials and life sciences, and various new combined techniques have been introduced and led to new discoveries mainly [3] at nano-to-micron scales throughout the last years.

In this contribution we present current advancement in femtosecond (fs) laser-equipped plasma FIB (PFIB)-SEM technology [4-5], multi-ion PFIB-SEM microscopes, in situ sample preparation and 3D data collection strategies together with micro x-ray computed tomography $(\mu \mathrm{CT})$ in the view of 3D CMT workflows. Applications of fs-laser in PFIB-SEM system in the workflow allows in excess of 1-mm size cross-sections in all dimensions, automated serial sectioning tomography (SST) and SEM recording of nanosized microstructural features. Further, oxygen PFIB works very efferently with polymeric and biological samples producing smooth, curtain-free surfaces allowing dozen of nm thick material removal in automated serial sectioning. The samples and RoI coordinates from $\mu \mathrm{CT}$ data are tracked in the PFIBSEM platform by a navigation software where $\mu \mathrm{CT}$ information from a visualization package and SEM images are co-registered. Finally, data from SST study is combined with $\mu$ CT results using the visualization and quantification research platform.

Here we present and discuss practical academic and industrial use-cases for such workflows. Specifically, we have investigated: (a) cracking in blown powder laser-deposited IN718 coupons produced from an atomized powder and high power 5kW laser melting; (b) sparsely distributed imperfections and defects in automotive fiber-reinforced polymer composite for filter casings; (c) nanoparticles and imperfections in conductive polymers used in the automotive fuel tank industry. 
References:

[1] B Winiarski at al., Supplement of Microscopy and Microanalysis 152 (2017), p. S4-S9.

[2] B Winiarski at al., Microscopy and Microanalysis 23 (S1/2017), p. 342-343.

[3] TL Burnett et al., Scientific Reports 4 (2014), p. 4711.

[4] SJ Randolph et al., Journal of Vacuum Science \& Technology B36 (2018), p. 06JB01.

[5] MP Echlin and A Mottura et al., Review of Scientific Instruments 83 (2012), p. 023701.

[6] B Winiarski at al., Microscopy and Microanalysis 24 (S1/2018), p. 366-367.

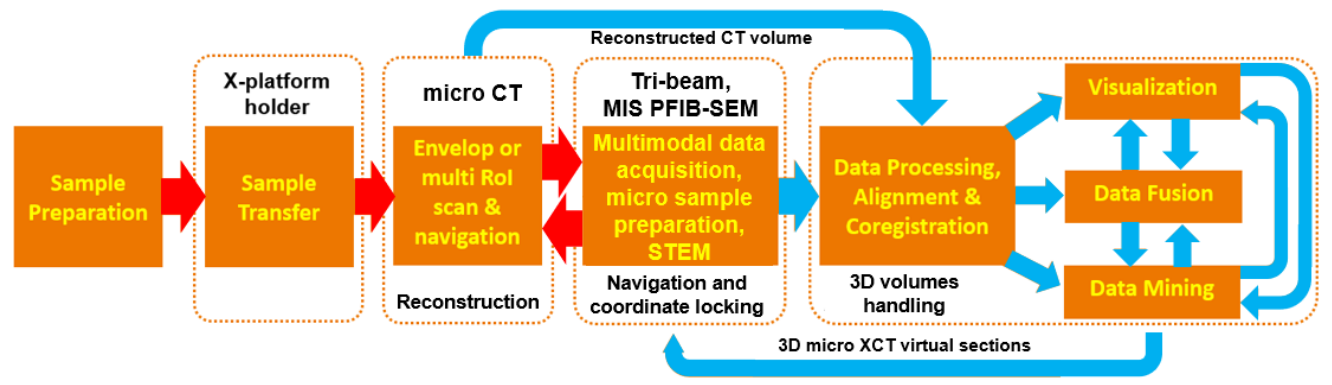

Figure 1. Diagram shows the correlative microscopy/tomography (CMT) workflow for material science. Red arrows indicate sample transfer, while blue arrows show data transfer. CMT is based on dedicated suite of instruments and software.
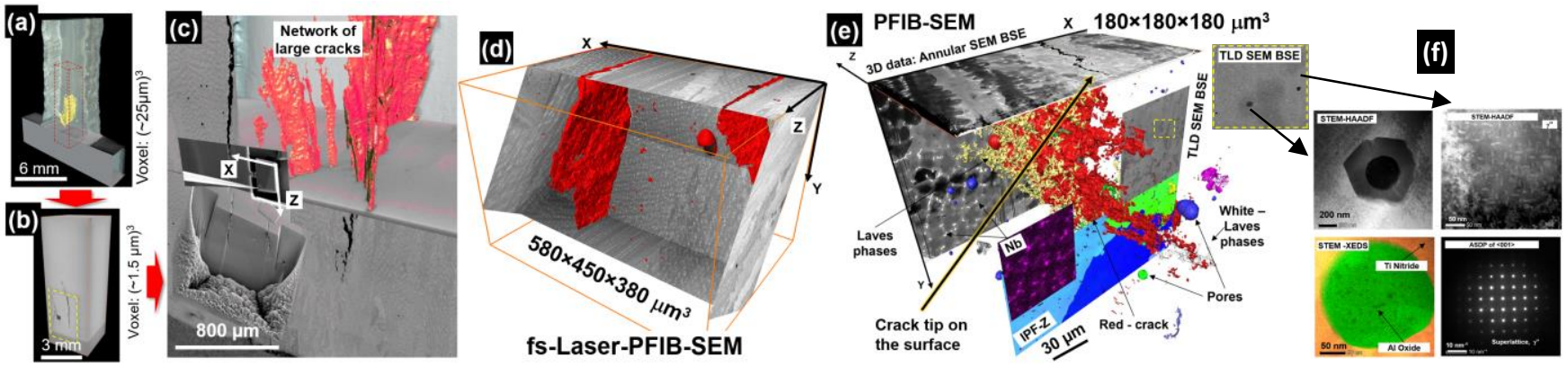

Figure 2. Images show results of CMT applied to IN718 additively manufactured specimen. (a) After envelop $\mu \mathrm{CT}$ scan; (b) After high resolution $\mu \mathrm{CT}$ scan; (c) visualization of combined multimodal data; (d) tri-beam serial sectioned material volume, segmented and visualized. (e) the crack tip segmented and visualized, including TEM study (f), after [6].

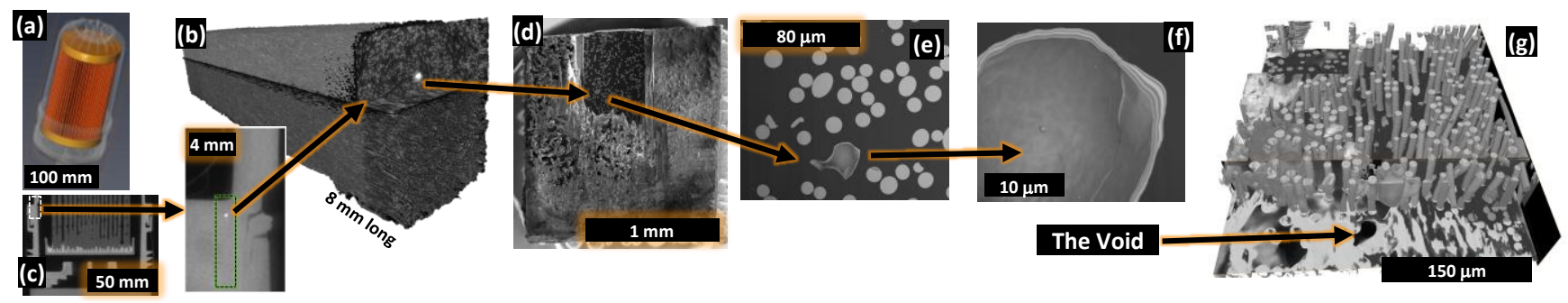

Figure 3. Images show correlative study of glass fiber reinforced polymer composite with defects in oil filter casings. (a) visualization of $\mu \mathrm{CT}$ shows inner details of the filter; (b) $\mu \mathrm{CT}$ volume rendering with RoI (particle?); (c) $\mu \mathrm{CT}$ sections showing details of the casing (d-f) SEM images taken after serial sectioning with oxygen PFIB showing the RoI (void with multilayer coating); (g) shows volume rendering of the serial sectioned volume containing the void. 